\title{
ESTUDIO PARA LA IMPLANTACIÓN \\ DE UN SERVICIO DE CONGELAMIENTO \\ DE CONCHAS de ABANICO EN el PeRÚ \\ MEDIANTE LA INSTALACIÓN DE UN EQUIPO \\ DE CONGELACIÓN CRIOGÉNICA
}

\author{
Alberto CARlos \\ https://orcid.org/0000-0002-6528-1929 \\ MARCElo Morán* \\ https://orcid.org/0000-0002-2984-531X \\ Universidad de Lima, Perú
}

Recibido: 25 de mayo del 2020 / Aprobado: 13 de agosto del 2020

doi: https://doi.org/10.26439/ing.ind2020.n039.4919

RESUMEN: Este artículo exhibe los resultados de una investigación enfocada en la viabilidad técnica y comercial para el servicio de congelamiento de conchas de abanico, utilizando el método de congelamiento rápido con nitrógeno. Fueron obtenidos a partir de la identificación de la demanda del proyecto, diseño del servicio, ingeniería del servicio y análisis económico.

Palabras clave: concha de abanico / congelamiento rápido / nitrógeno / plantas de congelación / servicio / congelación criogénica

\section{A STUDY FOR INTRODUCING A SCALLOP FREEZING SERVICE IN PERU THROUGH THE INSTALLATION OF A CRYOGENIC FREEZING EQUIPMENT}

ABSTRACT: This article shows the results of a research focused on the commercial and technical viability of a scallop freezing service using the individual quick freezing (IQF) method with nitrogen. The results were obtained from the identification of the project demand, service design, service engineering and economic analysis.

Keywords: scallops / quick freezing / nitrogen / freezing plant / service / cryogenic freezing

\footnotetext{
*Correos electrónicos: 20130241@aloe.ulima.edu.pe, 20132040@aloe.ulima.edu.pe
} 


\section{INTRODUCCIÓN}

En los últimos años, las exportaciones de conchas de abanico han sufrido una caída significativa. Según Las conchas de abanico, las pepitas de oro del mar peruano (2017), esto se debe a que áreas de la bahía de Sechura, donde se encuentra la mayor producción de conchas de abanico, no logran cumplir las exigencias de la Unión Europea. Por lo tanto, han sido retiradas del Listado Oficial de Áreas de Producción de Moluscos Bivalvos autorizadas por la Unión Europea, lo cual ha disminuido la exportación de este producto gradualmente desde el año 2014, como se muestra en la tabla 1.

Actualmente, existen varios métodos para el congelado. Por ejemplo, el método por aire forzado donde "usan corrientes de aire a bajas temperaturas y a gran velocidad" (Ballesteros, 2013, p. 30). Sin embargo, es más lento que los otros métodos y causa excesiva pérdida de agua; su aplicación es mayormente usada en frutas y verduras. También existe el método de contacto directo que usa amoniaco o freón como agente refrigerante. "Este sistema consiste en varias placas de paredes dobles, y en su interior se encuentra circulando un refrigerante. El alimento es puesto entre las placas, las cuales presionan el alimento por medio de un sistema hidráulico ligero" (Rodríguez et al., 2007, p. 103). Sin embargo, el amoniaco tiene un olor fuerte que "puede ser dañino si se inhala en concentraciones elevadas [...] Los efectos de la inhalación del amoniaco varían desde la irritación hasta severas lesiones respiratorias, con posibles fatalidades si existe una concentración mayor" (Guevara, 2015, p. 36), son inflamables cuando hay presencia de elementos como el cloro, mercurio y a altas temperaturas se descompone en hidrogeno y nitrógeno, que al mezclarse con el oxígeno forma las condiciones para una ignición o explotación. Por otro lado, el uso del freón, compuesto por clorofluorocarbonos, según la normativa europea F-Gas ha sido restringido, pues "los clorofluorocarbonos (CFC) contribuyen en gran manera a la destrucción de la capa de ozono" (Hernández, 2016, p. 7).

Ante esos problemas identificados, se realizó el estudio para la implantación de un servicio de congelamiento con nitrógeno utilizando el método rápido (IQF - Individual Quick Freezing, por sus siglas en inglés), ya que el nitrógeno líquido puede trabajar en un rango de temperatura de $-60^{\circ} \mathrm{C}$ a $-120^{\circ} \mathrm{C}$, lo que permite una congelación rápida y la formación de cristales más pequeños en el producto a congelar y "obtendrá las ventajas de ganar tiempo de vida deteniendo la maduración y mantener en perfectas condiciones" (Loaiza et al., 2016, p. 25). Por lo tanto, para los productores disminuye el riesgo al exportar y les permitiría reducir costos en el flete, ya que tiene como opción la vía marítima. Además, no tiene efectos toxicológicos ni es un gas inflamable, y según la clasificación CEE, no figura como sustancia peligrosa al ser un gas seco e inerte. Sin embargo, si no hay una buena ventilación existe el riesgo de asfixia por desplazamiento de oxígeno y si se encuentra a temperaturas frías, como $-196^{\circ} \mathrm{C}$, puede provocar quemaduras si hay contacto. Por lo tanto, dentro del plan de seguridad la implementación de equipos de protección personal (EPP) es fundamental. 
Tabla 1

Data histórica de exportaciones de conchas de abanico en toneladas

\begin{tabular}{lllllllllll}
\hline Producto & 2008 & 2009 & 2010 & 2011 & 2012 & 2013 & 2014 & 2015 & 2016 & 2017 \\
\hline $\begin{array}{l}\text { Conchas } \\
\text { de abanico }\end{array}$ & 6924 & 9436 & 12612 & 10462 & 5820 & 15121 & 12332 & 6178 & 4372 & 3262 \\
\hline
\end{tabular}

Fuente: Organización de las Naciones Unidas para la Alimentación y la Agricultura (2018)

\section{METOdOLOGÍA}

Para determinar la viabilidad técnica y comercial, se siguió la siguiente metodología de trabajo:

1. Descripción del servicio. Se identifican las áreas involucradas, como cliente, servicio al cliente, producción, distribución, y se determina los procedimientos a seguir para el desarrollo del servicio de congelamiento.

2. Estudio de mercado. Se hace un análisis del sector industrial para crear estrategias durante su funcionamiento. Después, se busca la demanda de proyecto considerando las exportaciones del Perú y el consumo de conchas de abanico en el mundo como las variables.

3. Ingeniería del servicio. Se realiza primero un cuadro comparativo entre las tecnologías de congelamiento y sus agentes refrigerantes disponibles en el mercado. Una vez determinada la mejor tecnología, se explica el proceso de obtención de nitrógeno y el funcionamiento de la cámara frigorífica. Finalmente, se diseña el plan de seguridad y el plano del servicio.

4. Análisis económico. En esta etapa se identifica el total de inversión y se realiza el flujo económico para evaluar la rentabilidad del servicio.

\section{RESULTADOS}

El proyecto consiste en ofrecer el servicio de congelamiento rápido con la implementación de una planta productora de nitrógeno para productos de exportación, con la finalidad de conservar sus propiedades y mantener su calidad.

El servicio se inicia cuando el cliente se contacta con el servicio de atención de la empresa para congelar su producción de conchas de abanico. El servicio de atención al cliente recibe la solicitud y verifica si hay capacidad de atención en la planta. Si no la hay, se comunica la no disponibilidad; en caso contrario, se envía una proforma al cliente. El cliente evalúa la proforma; si acepta, envía las conchas de abanico y el área de producción se encargará de recibirlas y desempacarlas para iniciar el proceso de congelamiento. Una vez terminado el proceso, se empaca y se estampa según la marca del cliente, para enviar 
al área de distribución donde el cliente recogerá su pedido y, finalmente, hará el pago del servicio. En la figura 1 se muestra el flujograma del servicio propuesto.

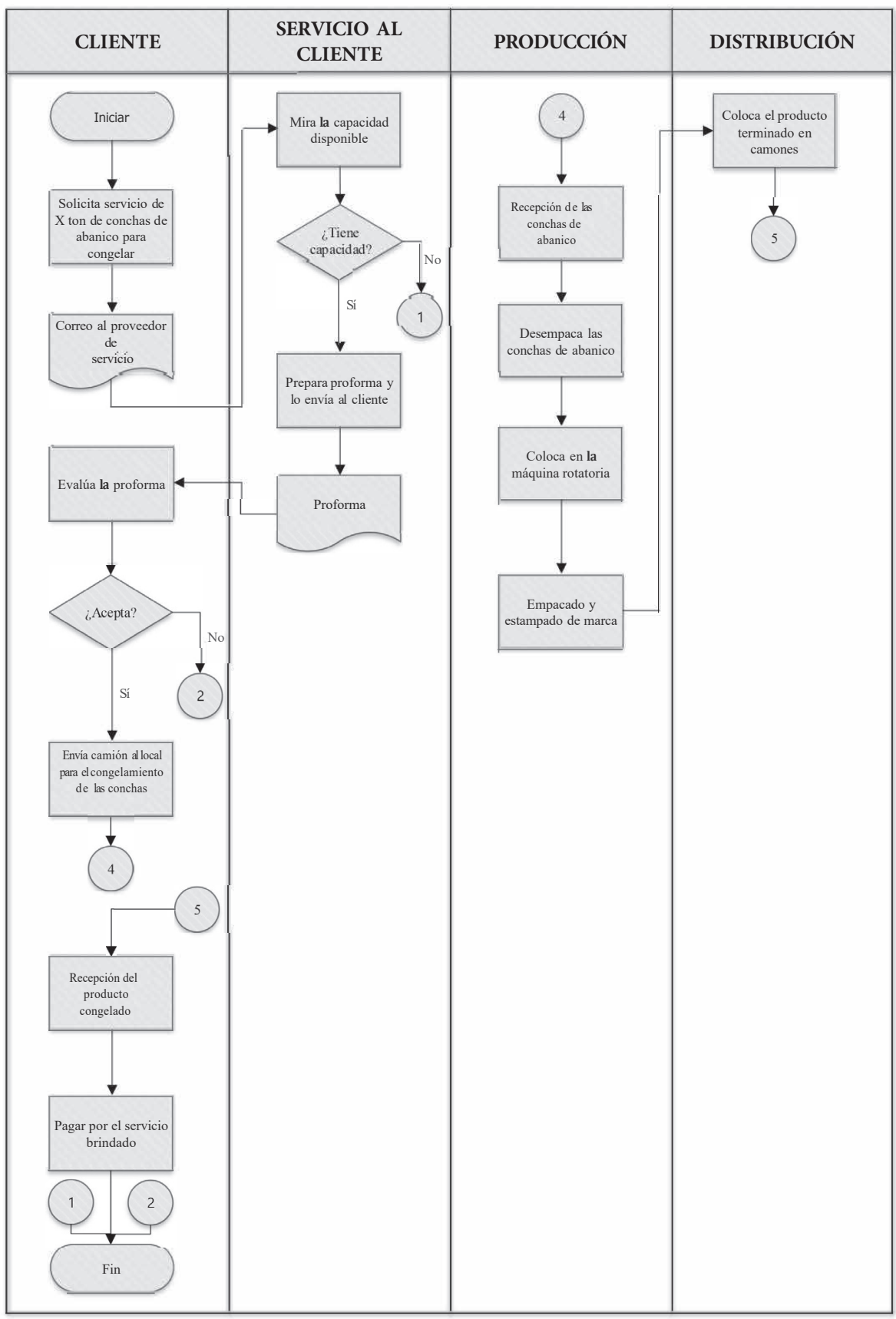

Figura 1. Flujograma para el servicio de congelamiento de conchas de abanico Elaboración propia 
Adicionalmente al servicio de congelado, con la finalidad de alcanzar la fidelización del cliente se ofrecerá lo siguiente:

- Se brinda el empaquetado del producto con la finalidad de dejarlo listo para que el cliente pueda exportar sin problemas. El servicio incluye el etiquetado con el nombre o marca de la empresa que adquiera la prestación.

- Como servicios complementarios, se ofrecerá garantía. Se asegura en devolver el producto congelado de acuerdo con sus especificaciones y se ofrecerá crédito hasta que su producto sea exportado, para demostrar al cliente la confiabilidad del servicio.

Para lograr un posicionamiento en el sector industrial de congelamiento de alimentos y realizar una estrategia competitiva eficiente se realizó el análisis de las cinco fuerzas de Porter mostrado en la figura 2.

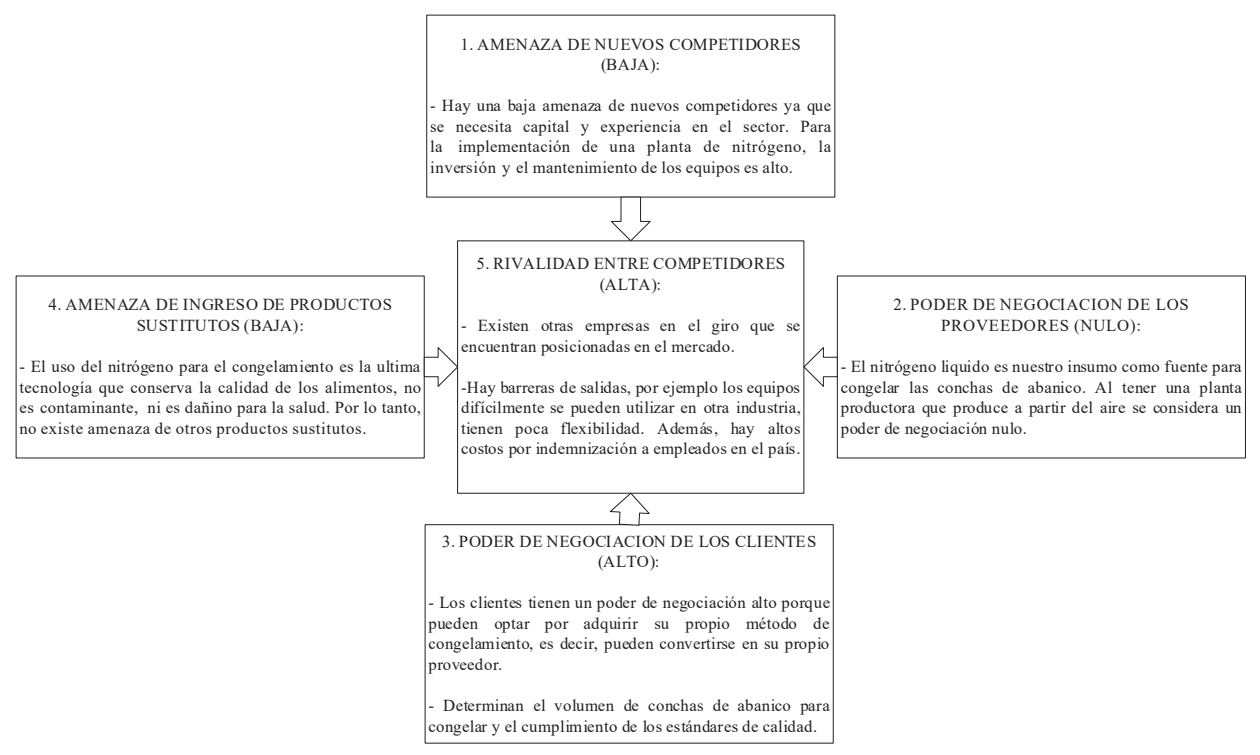

Figura 2. Análisis de las cinco fuerzas de Porter

Elaboración propia

En el estudio de mercado se han considerado la exportación de conchas de abanico y la demanda a analizar, ya que todas estas tienen que estar congeladas para venderse en los mercados internacionales, según el Decreto Supremo n. ${ }^{\circ} 07$ (2004) y el Decreto Supremo n. 004 (2018), controlado por el Organismo Nacional de Sanidad Pesquera (SANIPES). Como variable independiente a las exportaciones, se ha considerado el consumo mundial. 
Según el Banco Mundial (2013), la tendencia de consumo humano de la acuicultura tiene un crecimiento anual de $0,3 \%$ en promedio global. Además, existe un gran aumento en las inversiones para la acuicultura, sobre todo en tecnologías para mejorar la productividad, que incluyen el uso del agua de manera responsable, el mejoramiento genético en crías, las prácticas en los criaderos y la innovación en la alimentación de los peces (Unión Industrial Bahía Blanca, 2017, párr. 10).

Para la proyección de la demanda se analizó la data histórica de las exportaciones de conchas de abanico mencionada en la tabla 1 y se definió como la variable dependiente para la proyección. No se consideró para la proyección el año 2012, debido a una alta tasa de mortalidad por la presencia de marea roja, ni los años 2014 al 2017, ya que, ocurrieron eventos atípicos que afectaron la producción de las conchas de abanico como el fenómeno El Niño y el mal aprovisionamiento de semillas de conchas de abanico.

También, se analizó la data histórica del consumo de conchas de abanico, como se puede observar en la tabla 2, y se definió como la variable independiente.

Tabla 2

Consumo mundial de conchas de abanico en toneladas

\begin{tabular}{lcccccccc}
\hline Producto & 2008 & 2009 & 2010 & 2011 & 2012 & 2013 & 2014 & 2015 \\
\hline $\begin{array}{l}\text { Conchas } \\
\text { de abanico }\end{array}$ & 116793 & 122649 & 132078 & 136065 & 124091 & 154820 & 158884 & 173752 \\
\hline
\end{tabular}

Fuente: Organización de las Naciones Unidas para la Alimentación y la Agricultura (2018)

Como se mencionó en la "Definición y análisis de mercado", el consumo de los productos marinos tendrá un crecimiento de $0,3 \%$ anual. Por la tanto, se tiene la siguiente proyección con un ajuste lineal del 2018 al 2024, como se muestra en la figura 3, cuyos datos corresponden a la tabla 3.

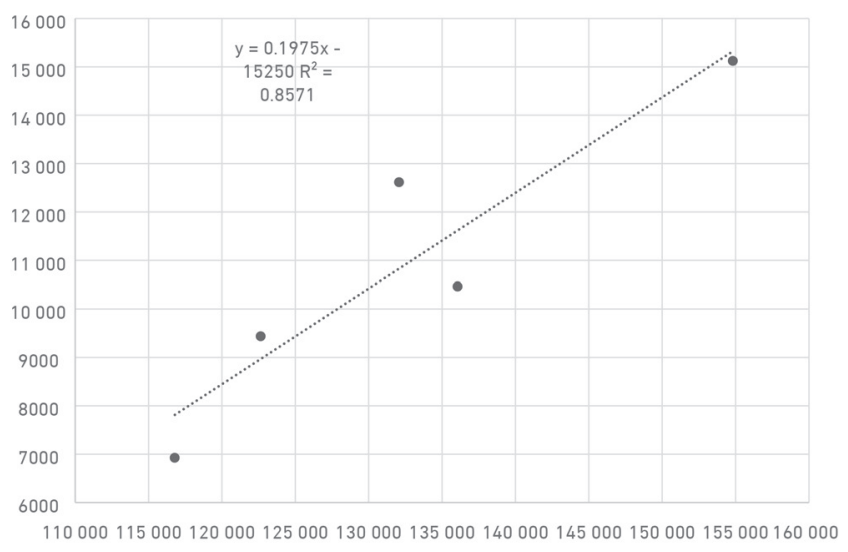

Figura 3. Tendencia de la demanda en toneladas

Elaboración propia 
Tabla 3

Proyección de la demanda en toneladas

\begin{tabular}{lc}
\hline Año & Producción de nitrógeno (T) \\
\hline 2018 & 19375,79 \\
2019 & 19479,67 \\
2020 & 19583,86 \\
2021 & 19688,36 \\
2022 & 19793,17 \\
2023 & 19898,30 \\
2024 & 20003,75 \\
\hline
\end{tabular}

Elaboración propia

Para este cálculo se analizaron tres componentes: la segmentación de la producción de conchas de abanico en el Perú; la intención e intensidad, que se determinaron mediante encuestas a los productores de conchas de abanico; la competencia y su participación de mercado.

Según la evaluación de microlocalización, se obtuvo que Sechura es el lugar apropiado para establecer las operaciones del congelado. Según Las conchas de abanico, las pepitas de oro del mar peruano (2018), en la bahía de Sechura (Piura) se produce un $80 \%$ de la producción total de conchas de abanico en el país.

Las empresas identificadas y su respectiva participación en el mercado son las siguientes: IPRISCO S.A.C., abarca un $60 \%$ del procesamiento actual de congelamiento, PERU PEZ S.A. y GAM CORP. procesan un $15 \%$ cada una, y UNITED OCEANS S.A. un $10 \%$. Al considerar que esta sería una empresa que recién se ha formado, la participación en el mercado sería la menor, es decir, el $10 \%$.

Además, mediante encuestas realizadas a productores de conchas de abanico se pudo conocer la intensidad y la intención del servicio, lo cual dio como resultado un factor de corrección de 0,49 (intensidad x intención). Con estos datos obtenidos en el estudio se tiene la demanda de proyecto que se muestra en la tabla 4. 
Tabla 4

Demanda del proyecto en toneladas

\begin{tabular}{lcccccc}
\hline Año & $\begin{array}{c}\text { Producción } \\
\text { de nitrógeno } \\
(\mathrm{T})\end{array}$ & $\begin{array}{c}\text { Segmentación } \\
\text { (Sechura 80 \%) }\end{array}$ & $\begin{array}{c}\text { Factor de } \\
\text { corrección } \\
\text { (Intención } x \\
\text { Intensidad) }\end{array}$ & $\begin{array}{c}\text { Demanda } \\
\text { Objetivo } \\
(\mathrm{T})\end{array}$ & $\begin{array}{c}\text { Participación } \\
(10 \%)\end{array}$ & $\begin{array}{c}\text { Demanda } \\
\text { del } \\
\text { proyecto } \\
\text { (T) }\end{array}$ \\
\hline 2018 & 19375,79 & $80 \%$ & 0,49 & 7595,31 & 0,10 & 759,53 \\
2019 & 19479,67 & $80 \%$ & 0,49 & 7636,03 & 0,10 & 763,60 \\
2020 & 19583,86 & $80 \%$ & 0,49 & 7676,87 & 0,10 & 767,69 \\
2021 & 19688,36 & $80 \%$ & 0,49 & 7717,84 & 0,10 & 771,78 \\
2022 & 19793,17 & $80 \%$ & 0,49 & 7758,92 & 0,10 & 775,89 \\
2023 & 19898,30 & $80 \%$ & 0,49 & 7800,14 & 0,10 & 780,01 \\
\hline 2024 & 20003,75 & $80 \%$ & 0,49 & 7841,47 & 0,10 & 784,15 \\
\hline
\end{tabular}

Elaboración propia

El servicio usará la tecnología de congelamiento rápido con nitrógeno líquido. "El principal efecto de la congelación sobre la calidad de los elementos es el daño que ocasiona en las células el crecimiento de cristales de hielo" (Umaña, 2016, p. 27), es decir, mientras los cristales de congelamiento sean más pequeños -y por ende el congelamiento más rápido-, los tejidos de las conchas no se dañan y sus propiedades se conservan. Es por ello que se considera:

- Congelamiento por aire forzado. No se considera oportuno debido a que el congelamiento es más lento; por lo tanto, los cristales que se forman son más grandes y el producto pierde propiedades organolépticas.

- Congelamiento usando amoniaco o freón. Si bien tiene un buen consumo energético, los cristales formados a comparación del uso del nitrógeno son grandes y existe mayor pérdida tanto en el peso de congelado y descongelado.

- Congelamiento usando nitrógeno. Se considera esta técnica como la óptima; con esta tecnología tenemos una rapidez del congelamiento, provocando así cristales menores y por ende una mejor conservación de las propiedades organolépticas. Por otro lado, al momento de la descongelación, en comparación de otras tecnologías, se puede descongelar la cantidad deseada y no todo un bloque de producto.

En la tabla 5 se hace una comparación más detallada entre los elementos nitrógeno, amoniaco y freón para el congelamiento. 
Tabla 5

Cuadro comparativo entre elementos para la congelación de alimentos

\begin{tabular}{lcc}
\hline Variables & Amoniaco / Freón & Nitrógeno \\
\hline Temperatura & $\left(-20{ }^{\circ} \mathrm{C}\right) /\left(-50^{\circ} \mathrm{C}\right)$ & $\left(-196^{\circ} \mathrm{C}\right)$ \\
Cristales formados & Grandes & Pequeños \\
Perdida de peso congelado \% & 0,5 a 0,24 & 0,11 \\
Perdida de peso descongelado \% & 0,8 a 0,7 & 0,58 \\
Tiempo (min) (en un producto de $200 \mathrm{~g})$ & 12,8 & 8,2 \\
Espacio $\mathrm{m}^{2}$ (procesando $\left.2000 \mathrm{~kg} / \mathrm{h}\right)$ & 62 & 19 \\
\hline
\end{tabular}

Fuente: Air Products (2016)

Elaboración propia

\subsection{Funcionamiento de una planta de nitrógeno}

Para poder ofrecer el servicio, se necesita la producción de nitrógeno líquido, el cual se obtendrá mediante el método de destilación que se detallará a continuación y está representado en un diagrama de flujo como se observa en las figuras 4 y 5 .

El aire atmosférico pasa por una filtración preliminar para eliminar todas las partículas gruesas, como el polvo, que puedan poner en riesgo la integridad de los compresores. Luego, el aire se comprime hasta una presión de 5 bares, donde el aire se calentará y deberá ser enfriado hasta una temperatura de $20^{\circ} \mathrm{C}$. Luego, el aire se prenfría en un chiller y se vuelve a condensar en un tanque de aire (Cerrada, 2017, pp. 22-33). A continuación, pasa a un secador filtro molecular, donde se desecha, gases como $\mathrm{CO}_{2}$, hidrocarburos y CO. Posteriormente, la corriente de aire se vuelve a comprimir y pasa a la torre de destilación de alta presión; ahí, el proceso consiste en la rectificación de la temperatura y masa y se obtiene la fase gaseosa de nitrógeno; luego, pasa a una torre de destilación de baja presión en esta etapa se enfría, permitiendo la licuación del nitrógeno mediante un booster. 


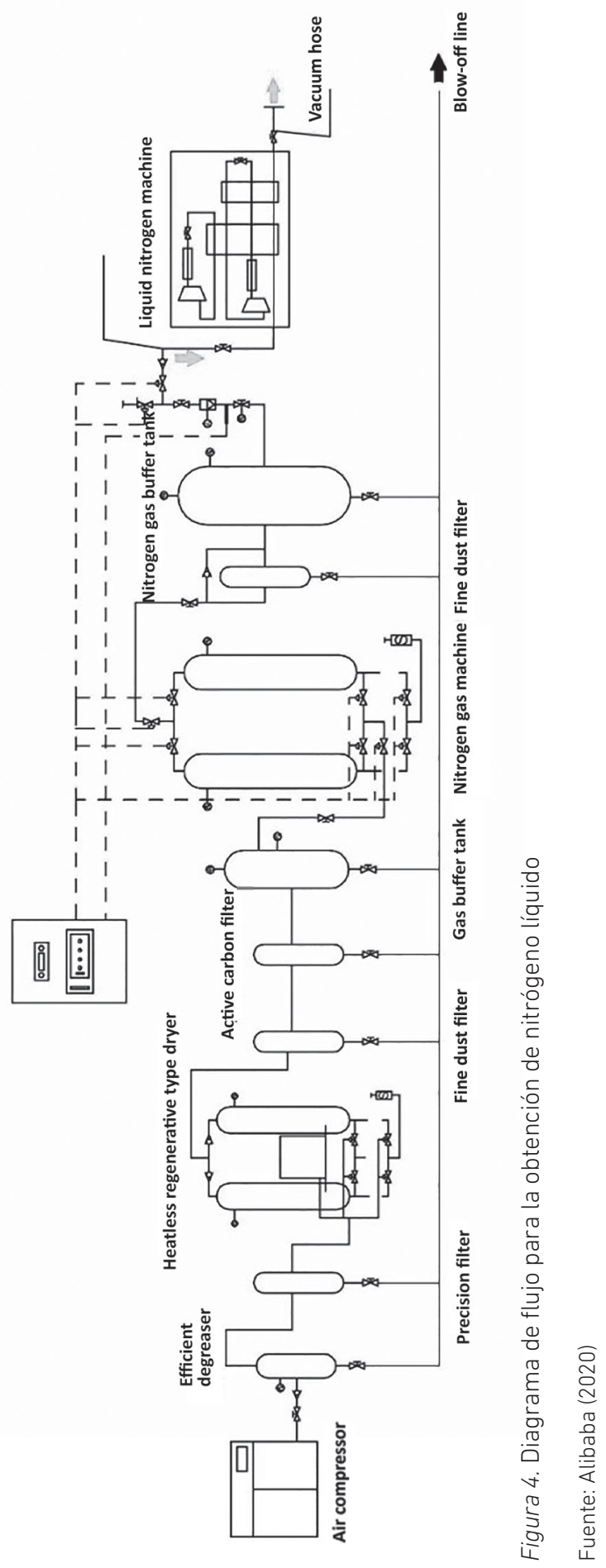




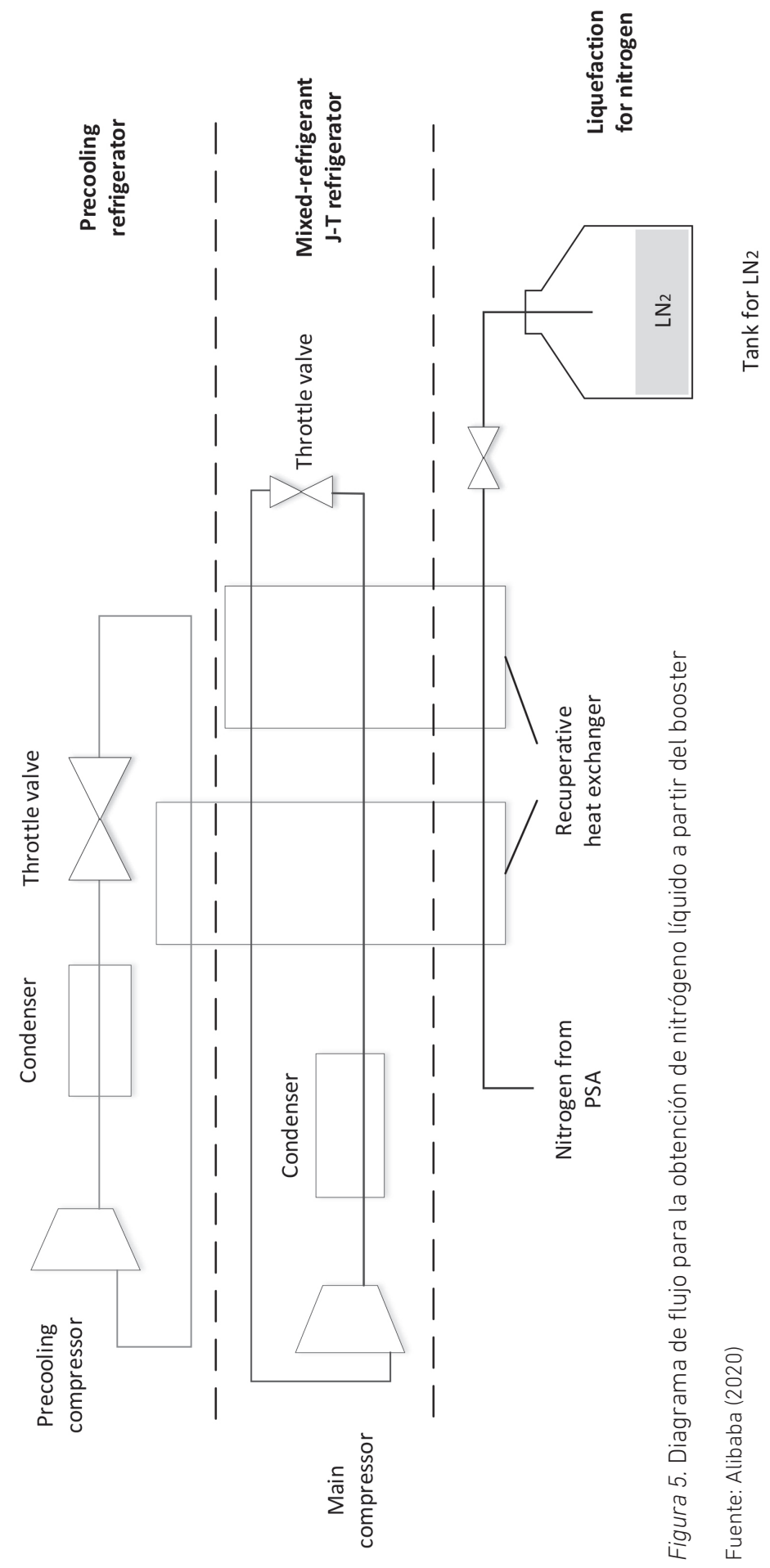


Después, el nitrógeno líquido producido pasará a una máquina rotatoria o túnel de congelación, cuya función será bañar a las conchas de abanico y de esta forma producir su congelamiento inmediato (método IQF). Este túnel utilizará la tecnología de congelación por aspersión y está compuesto por varias cintas que evitan que los alimentos se peguen entre sí. La caída continuada del producto crea un óptimo resultado del congelamiento IQF. Interiormente contiene ventiladores que dispersan el nitrógeno líquido vaporizado para un mejor rendimiento y pulverizadores que se encargan de cubrir el producto con nitrógeno líquido. Este tipo de diseño permite congelar desde productos marinos hasta frutas o verduras.

Para tener un servicio con atención continua y una política de cero accidentes, es necesario hacer un análisis de seguridad. El estudio inició con el desarrollo de un mapa de riesgos de la planta (tabla 6), donde se identificaron el factor de riesgo, su ubicación y su fuente, para establecer una medida preventiva.

Posteriormente, se identificaron los tipos de fuego (tabla 7) que se pueden ocasionar en la planta y se definen las formas de actuar en caso de que ocurran.

Por último, se realizó la matriz de identificación de peligros y riesgos (tabla 8) para establecer las medidas de control para cada peligro identificado. A continuación, se muestran las tablas mencionadas previamente:

Tabla 6

Mapa de riesgos en el servicio de congelamiento

\begin{tabular}{|c|c|c|c|}
\hline Factor de riesgo & Ubicación & Fuente & Medida preventiva \\
\hline $\begin{array}{l}\text { Actividad de } \\
\text { mantenimiento }\end{array}$ & $\begin{array}{l}\text { Estación de } \\
\text { mantenimiento }\end{array}$ & Maquinas del proceso & $\begin{array}{l}\text { Capacitación y utilización } \\
\text { de EPP`S }\end{array}$ \\
\hline $\begin{array}{l}\text { Descarga de bandejas con } \\
\text { concha de abanico }\end{array}$ & Estación de recepción & Bandejas & $\begin{array}{l}\text { Capacitación sobre los } \\
\text { peligros ergonómicos. }\end{array}$ \\
\hline $\begin{array}{l}\text { Llenado con hielo a las } \\
\text { bandejas }\end{array}$ & Estación de pesado & Hielo & $\begin{array}{l}\text { Proporcionar guantes } \\
\text { termoaislantes }\end{array}$ \\
\hline $\begin{array}{l}\text { Congelamiento de conchas } \\
\text { de abanico }\end{array}$ & Estación de congelado & $\begin{array}{l}\text { Ambiente en baja } \\
\text { temperatura }\end{array}$ & $\begin{array}{l}\text { Proporcionar vestimenta } \\
\text { contra baja temperatura }\end{array}$ \\
\hline $\begin{array}{l}\text { Colocación de producto } \\
\text { congelado en bolsas }\end{array}$ & Área de envasado & $\begin{array}{l}\text { Conchas de abanico } \\
\text { congeladas }\end{array}$ & $\begin{array}{l}\text { Proporcionar guantes } \\
\text { aislantes }\end{array}$ \\
\hline Descarga eléctrica & $\begin{array}{l}\text { Lugares con } \\
\text { maquinarias }\end{array}$ & Maquinas en general & $\begin{array}{l}\text { Capacitación y colocar } \\
\text { conexiones a tierra }\end{array}$ \\
\hline Piso mojado & Toda la planta & $\begin{array}{l}\text { Hielo y nitrógeno } \\
\text { liquido }\end{array}$ & $\begin{array}{l}\text { Proporcionar botas contra } \\
\text { agua }\end{array}$ \\
\hline
\end{tabular}

Elaboración propia 


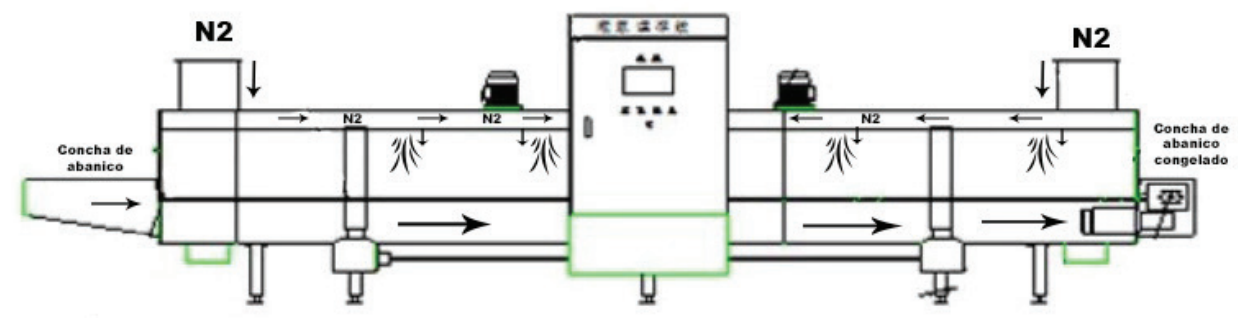

Figura 6. Funcionamiento de túnel de congelamiento

Elaboración propia

Tabla 7

Tipos de fuego

\begin{tabular}{cll}
\hline Tipo & Material de origen & Forma de actuación \\
\hline B (Gases líquidos inflamables) & Combustible & $\begin{array}{l}\text { Utilizar dióxido de carbono } \\
\text { o espuma fluoroproteinica }\end{array}$ \\
C (Materiales energizados) & Máquinas, cableado & Utilizar gas carbónico $\mathrm{CO}_{2}$ \\
\hline
\end{tabular}

Elaboración propia

Las capacitaciones serán al inicio de cada jornada y se usarán de manera permanente equipos de protección personal tales como:

- Guantes termoaislantes.

- Botas de hule o calzado impermeable.

- Vestimenta térmica con reflectores.

- Orejeras.

- Lentes de seguridad.

Para el análisis económico se identificó que el total de la inversión es de S/ 1716 046,50, como se puede ver en la tabla 9. Los activos intangibles incluyen la licencia de funcionamiento, contingencias, capacitaciones, gasto de remodelación de terreno. Los activos tangibles incluyen la maquinaria (planta de nitrógeno, túnel de congelación, estampadora), equipos, mobiliario de oficina y equipamiento. 


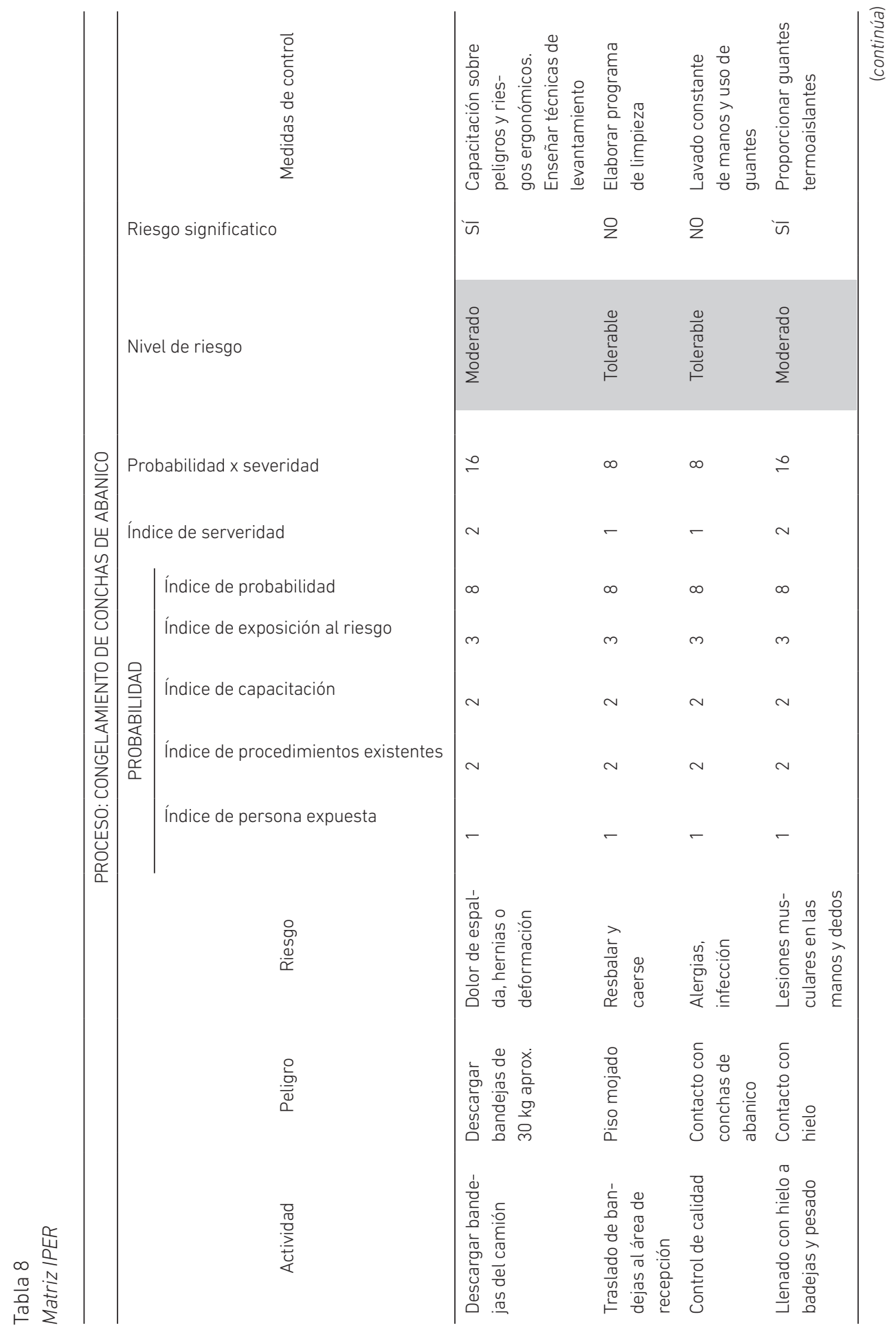


Estudio para la implantación de un servicio de congelamiento de conchas de abanico

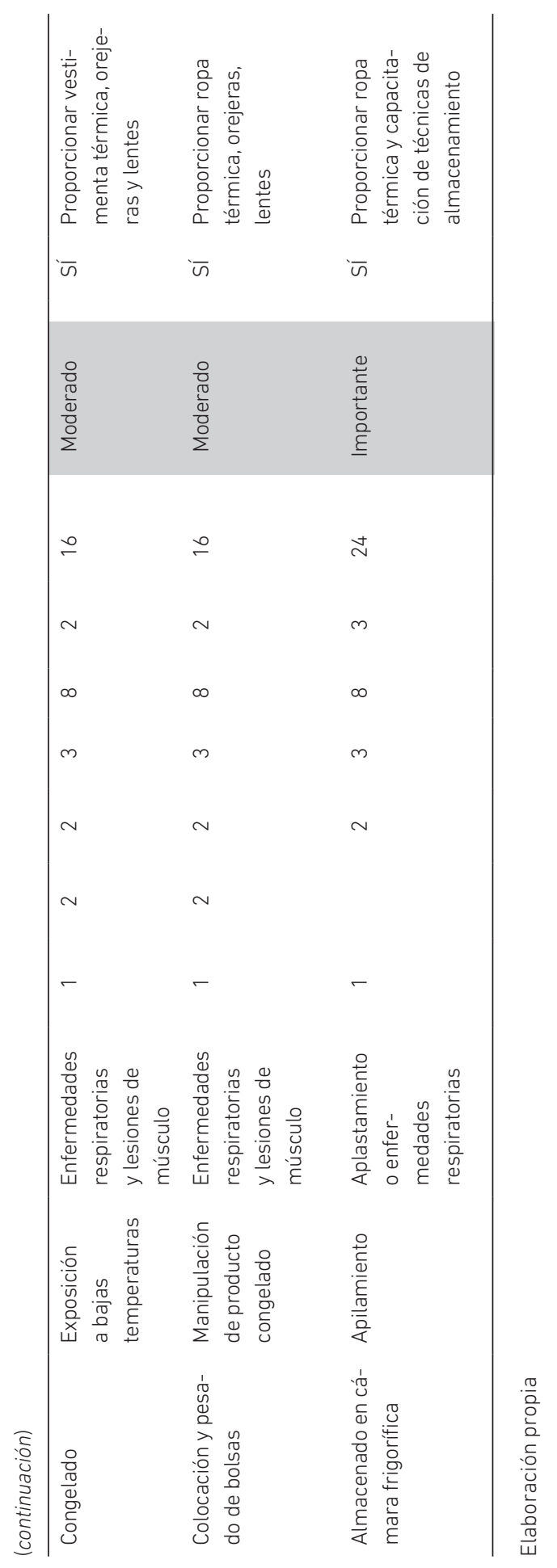


Tabla 9

Desagregado del total de inversión

\begin{tabular}{lr}
\hline \multicolumn{1}{c}{ Tipo } & \multicolumn{1}{c}{ Monto (S/) } \\
\hline Activos intangibles & 564832,38 \\
Activos tangibles (maquinaria & 1032930,32 \\
y equipos, mobiliaria) & \\
Capital de trabajo & 118283,80 \\
Total de inversión & 1716046,50 \\
\hline
\end{tabular}

Elaboración propia

Los ingresos se obtienen por tonelada congelada. El precio del servicio por tonelada es de $S / 2600$. Dentro de costos y gastos se consideró el costo de materia prima, costo de servicios y alquileres, y costo de personal. Para hallar el costo de oportunidad se utilizó el método CAPM (COK= Rf + beta (Rm-Rf)) y se consideraron los siguientes datos recolectados de Bloomberg, obteniendo el siguiente resultado:

$$
\begin{aligned}
& \mathrm{Rf}^{1}=5,78 \% \\
& \mathrm{~B}^{2}=1 \\
& \mathrm{Rm}^{3}=9,56 \%
\end{aligned}
$$

$$
\text { Cok }=5,78 \%+1(9,56 \%-5,78 \%)=9,56 \%
$$

Bajo este escenario, se realiza el flujo económico como se muestra en la tabla 10 y se obtienen los siguientes indicadores (ver tabla 11).

1 Tasa libre de riesgo.

2 Beta del activo.

3 Rentabilidad del mercado. 


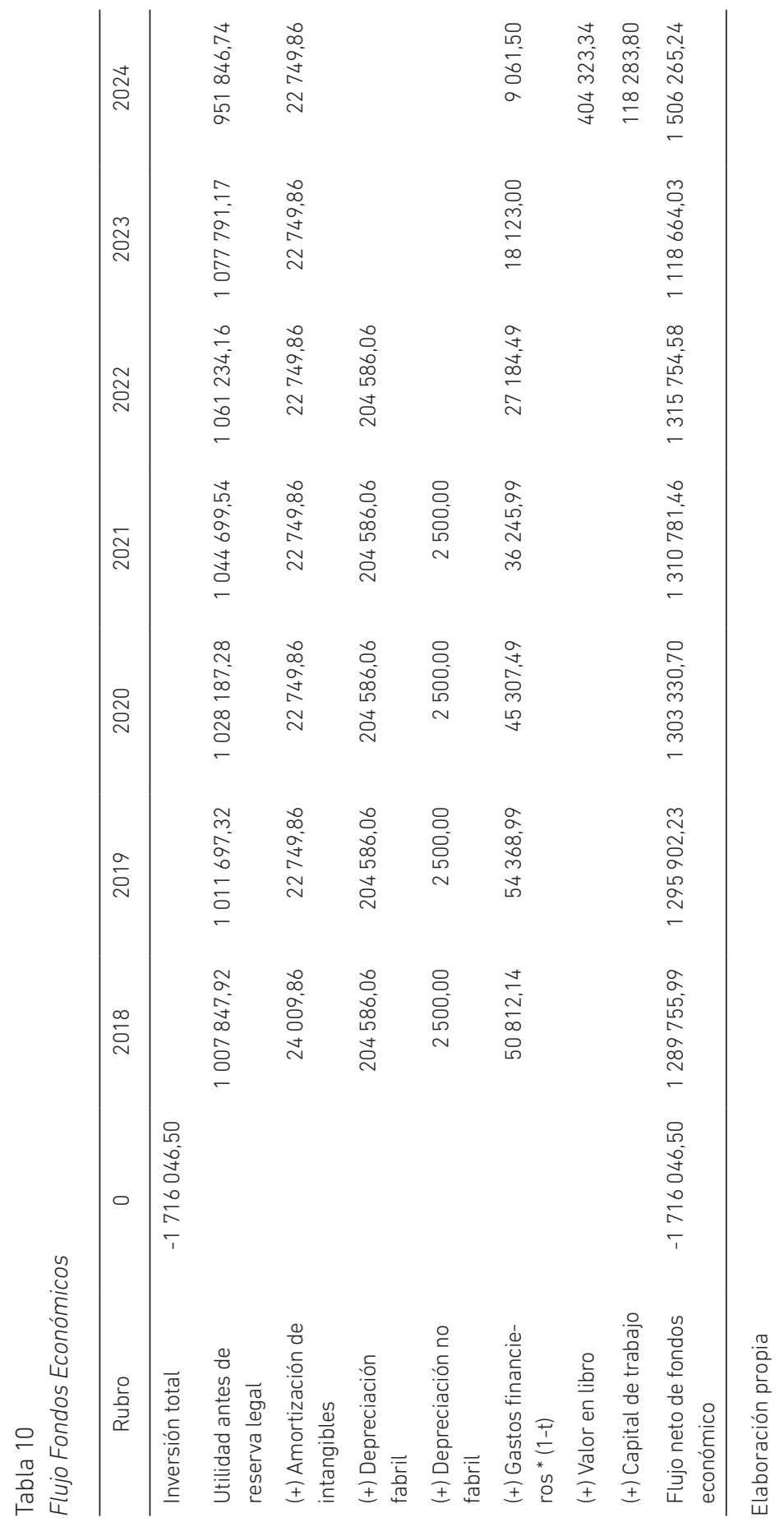


Tabla 11

Evaluación económica

\begin{tabular}{lr}
\hline Van económico & 4716877,17 \\
B/c & 3,75 \\
Tasa interna de retorno económico & $73,89 \%$ \\
Periodo de recuperación (años) & 1,50 años \\
\hline
\end{tabular}

Elaboración propia

El valor actual neto (VAN) es de S/ 4716877,17 , lo que significa que el proyecto es rentable. Además, el valor de la tasa interna de retorno (TIR) es de 73,89\%, al ser superior al COK calculado (9,56 \%); esto nos indica que el proyecto es aceptado por los inversionistas. Respecto del beneficio-costo, por cada sol invertido se gana S/ 3,75. Finalmente, el proyecto tiene siete años de horizonte y el periodo de recupero es de 1,50 años. Es decir, al segundo año se generan ganancias.

\section{CONCLUSIONES}

La implantación del servicio de congelamiento de conchas de abanico es viable comercialmente porque existe una demanda en crecimiento, ya que el consumo por los productos marinos está aumentando. Sin embargo, en ocasiones puede verse alterada por factores externos como el fenómeno El Niño o la aparición de bacterias que afecten la producción y conlleve a una caída de las exportaciones. Sin embargo, el proyecto es rentable al tener un VAN positivo, una tasa interna de retorno mayor al costo de oportunidad y un beneficio costo mayor a 1 .

También es viable técnicamente, porque existe la tecnología de congelamiento rápido (IQF) con nitrógeno que permite a los productores cumplir con los estándares al momento de exportar los productos congelados y la facilidad de obtener nitrógeno a través del ambiente, gracias a la planta que permite que el servicio funcione de manera continua y segura. Es decir, el riesgo de que se detenga la atención por ausencia de nitrógeno $o$ accidentes es poco probable.

\section{REFERENCIAS}

Air Products (8 de junio del 2016). Cryogenic vs Mechanical Food Freezing [video]. YouTube. Recuperado de https://www.youtube.com/watch?v=AHV6SuRhtY8\&t=33s

Alibaba (2020). Cryo Chains: 50L/hour Automatic Liquid nitrogen generator LN2 production machine for food processing plant. Recuperado de https://cryochains.en.alibaba. com/product/62322605467-818294438/50L_hour_Automatic_Liquid_ 
nitrogen_generator_LN2_production_machine_for_food_processing_plant. html?spm=a2700.icbuShop.41413.11.588fabfdxLLPdZ

Ballesteros, L. (2013). Enfriamiento por aire forzado. Mundo HVAC\&R, 8(98), 30-33. Recuperado de https://www.mundohvacr.com.mx/2013/06/junio-2013/

Cerrada Martínez, P. (2017). Diseño y análisis de una planta de separación de aire [tesis de grado, Universidad Politécnica de Madrid, España]. Repositorio institucional de la Universidad Politécnica de Madrid. Recuperado de http://oa.upm.es/48874/

Decreto Supremo n. 004 (2018). Modificación del artículo 14 de la Norma Sanitaria de Moluscos Bivalvos Vivos. Recuperado de https://www.gob.pe/institucion/ produce/normas-legales/191190-004-2018-produce

DecretoSupremon. ${ }^{\circ}$ 07(2004).NormaSanitariadeMoluscosBivalvosVivos. Recuperado de http://www.sanipes.gob.pe/normativas/6_DECRETOSUPREM0007-2004-PRO DUCE.pdf

Guevara Salgado, A. W. (2015). Desarrollo de un plan de seguridad y salud ocupacional para mantener controlada la fuga de amoniaco y la aplicación de plan de emergencia y contingencia en caso de fugas de amoniaco en empresa empacadora de camarón [tesis para obtener el título de magíster en Seguridad, Higiene Industrial Salud Ocupacional, Universidad de Guayaquil, Ecuador]. Repositorio institucional de la Universidad de Guayaquil. Recuperado de http://repositorio.ug.edu.ec/handle/ redug/20993

Hernández Torres, G.A. (2016). El ozono: Impacto del adelgazamiento de la capa de ozono en el territorio antártico [monografía para obtener el diplomado en Asuntos Antárticos, Universidad de Magallanes, Chile]. Repositorio institucional de la Universidad de Magallanes, Convenio de Desempeño "Gaia Antártica: Conocimiento y Cultura Antártica".

Las conchas de abanico, las pepitas de oro del mar peruano (21 de diciembre del 2018). Gestión. Recuperado de https://gestion.pe/economia/conchas-abanico-pepitas -oro-mar-peruano-253564-noticia/?ref=gesr

Loaiza, K.; Erazo, S. y Velez, C. (2016). Congelación de arveja empleando un sistema de congelación individual, IQF/Freezing peas using an individual freezing system, IQF. Vitae, 23(1), 330-334. Recuperado de https://search.proquest.com/openview/ ff47819597fb991d3b016a33322121d4/1?pq-origsite=gscholar\&cbl=1806352

Organización de las Naciones Unidas para la Alimentación y la Agricultura [FAO] (2018). Visión general del sector acuícola nacional. Recuperado de http://www.fao.org/ fishery/countrysector/naso_peru/es 
Rodríguez Martínez, V.; Cerón Carrillo, T. G. y Vázquez, A. M. (2007). Descripción y aplicaciones de equipos de congelación para la industria de alimentos. Temas Selectos de Ingenieria de alimentos, 1(1), 97-107. Recuperado de https://tsia.udlap.mx/ tsia-vol-1-no-1-ago-dic-2007/

Umaña Cerros, E. (2016). Congelación de alimentos por frío, 27-28. Recuperado de https:// es.slideshare.net/FUSADESORG/conservacion-af-1

Unión Industrial Bahía Blanca (2017). Acuicultura: actualidad, tendencias y proyecciones. Tiempo Industrial, 101-103.

World Bank (2013). Fish to 2030: prospects for fisheries and aquaculture, 44-45. Recuperado de http://documents.worldbank.org/curated/en/458631468152376668/Fish-to2030-prospects-for-fisheries-and-aquaculture

\section{BIBLIOGRAFÍA}

Acedo, J. D. y Flores, J. (2015). Efecto de la temperatura en respuestas fisiológicas de la concha de abanico Argopecten purpuratus. Revista Peruana de Biología, 22(3), 329-334. doi: http://dx.doi.org/10.15381/rpb.v22i3.11439.

Air Products (1 de febrero del 2016). ¿Cómo funciona la congelación criogénica y la congelación superficial de productos IQF? (congelación individual rápida) [video]. Recuperado de https://www.youtube.com/watch?v=kSBIppiLE6U

Air Products (5 de noviembre del 2015). ¿Por qué congelar con nitrógeno líquido? [video]. Recuperado de https://www.youtube.com/watch?v=1hj4nzfL8YM

García, R., Pinto, A., Rengel, J., Torres, J., González, J. y Pérez, N. (2017). Diseño de una estrategia de control difuso aplicada al proceso de ultra congelación de alimentos/ Design of fuzzy control strategy applied to food process deep freeze. Ingeniare: Revista Chilena de Ingeniería, 25(1), 70-84. Recuperado de https://scielo.conicyt. $\mathrm{cl} / \mathrm{pdf} /$ ingeniare/v25n1/0718-3305-ingeniare-25-01-00070.pdf

Morales Landeo, R. (1986). Cálculo y diseño de un equipo experimental para la congelación de alimentos mediante nitrógeno líquido [tesis para optar el título profesional de ingeniero mecánico, Universidad Nacional de Ingeniería, Perú]. Repositorio institucional de la Universidad Nacional de Ingeniería. Recuperado de http:// cybertesis.uni.edu.pe/handle/uni/2803

Morán Gálvez, M. y Carlos Cuadros, A.E. (2019). Estudio de prefactibilidad para la instalación de una planta de nitrógeno para el servicio de congelamiento IQF para conchas de abanico. [tesis para optar el título de ingeniero industrial, Universidad de Lima, Perú]. Repositorio institucional de la Universidad de Lima. 
Recuperado de http://repositorio.ulima.edu.pe/bitstream/handle/ulima/9619/ Moran_Galvez_Marcelo_Andre.pdf?sequence=6\&isAllowed=y

Rudolph Delgado, F. (1989). Análisis tecnológico del congelamiento con nitrógeno de productos marinos de consumo humano [tesis para optar el título profesional de ingeniero industrial, Universidad de Lima, Perú]. Repositorio institucional de la Universidad de Lima. Recuperado de http://repositorio.ulima.edu.pe/bitstream/handle/ ulima/9619/Moran_Galvez_Marcelo_Andre.pdf?sequence=6\&isAllowed=y

Sánchez Cuéllar, L. (2017). Análisis de la cadena de valor de la concha de abanico en la Bahía de Sechura [tesis para optar el título de ingeniero pesquero, Universidad Nacional Agraria La Molina, Perú]. Repositorio institucional de la Universidad Nacional Agraria. La Molina. Recuperado de http://repositorio.lamolina.edu.pe/ bitstream/handle/UNALM/3273/sanchez-cuellar-luis-alberto.pdf?sequence $=1 \&$ isAllowed $=\mathrm{y}$

Sanipes (2015). Plantas de procesamiento primario de moluscos bivalvos habilitadas, 2017, de SANIPES. Recuperado de http://www.sanipes.gob.pe/frontend/plantasbivalvos.php 